\title{
Evolving Intelligent Reflector Surface towards 6G for Public Health: Application in Airborne Virus Detection
}

\author{
Harun Šiljak, Senior Member, IEEE, Nouman Ashraf, Student Member, IEEE, Michael Taynnan \\ Barros, Member, IEEE, Daniel Perez Martins, Member, IEEE, Bernard Butler, Member, IEEE, Arman \\ Farhang, Member, IEEE, Nicola Marchetti, Senior Member, IEEE, Sasitharan \\ Balasubramaniam, Senior Member, IEEE
}

\begin{abstract}
While metasurface-based intelligent reflecting surfaces (IRS) are an important emerging technology for future generations of wireless connectivity in its own right, plans for the mass deployment of these surfaces motivate the question of their integration with other new and emerging technologies that would require such widespread deployment. This question of integration and the vision of future communication systems as an invaluable component for public health motivated our new concept of Intelligent Reflector-Viral Detectors (IR-VD). In this novel scheme, we propose deployment of intelligent reflectors with strips of receptor-based viral detectors placed between the reflective surface tiles. Our proposed approach encodes information of the presence of the virus by flicking the angle of the reflected beams, using time variations between the beam deviations to represent the messages. This information includes the presence of the virus, its location and load size. The paper presents simulations to demonstrate the encoding process that represents the number of virus particles that have bound to the IR-VD.
\end{abstract}

Index Terms-6G communications, Virus detectors, Airborne virus, Intelligent Reflector Surfaces, Molecular communications, COVID-19.

\section{INTRODUCTION}

$\mathbf{T}$ HE recent COVID-19 pandemic has shocked the world, with numerous countries around the world facing lockdowns that have affected the global economy.

Since this is a global challenge, a collaborative effort is required from scientists that goes beyond experts such as virologists, immunologists and public health experts. We join the anti-COVID efforts with a vision from next generation networks. While $6 \mathrm{G}$ networks mainly focus on high capacity. low latency and high reliability requirements for applications as diverse as pervasive connectivity and Industry 4.0, here we join the growing community [1] calling for significant applications of $5 \mathrm{G}$ and $6 \mathrm{G}$ in public health. In this paper, we propose a new solution where wireless communication

HŠ and NM are with CONNECT Centre, Trinity College Dublin, Ireland; NA, DPM, BB and SB are with the VistaMilk Centre, Walton Institute, Waterford Institute of Technology, Ireland; MTB is with the University of Essex, United Kingdom; AF is with Maynooth University, Ireland. This publication has emanated from research conducted with the financial support of Science Foundation Ireland (SFI) and the Department of Agriculture, Food and Marine on behalf of the Government of Ireland under Grant Number [16/RC/3835] - VistaMilk research centre, and is co-funded under the European Regional Development Fund for the CONNECT research centre (13/RC/2077). Corresponding author (HŠ): harun.siljak@tcd.ie systems can play a role in detecting the virus shed by infected individuals.

This is based on the use of Intelligent Reflective Surfaces (IRS) that have been proposed for reflecting, refracting and diffracting electromagnetic waves in the mm-wave and terahertz spectrum. IRS [2], [3], [4] have been proposed to counter challenges of reliable communication at these wavelengths, by redirecting and reflecting beams to the mobile devices, to ensure non-LoS signal coverage. The introduction of IRS will require infrastructure changes, where this could come in the form of wallpapers that integrate electronics and nanotechnology to ensure compactness, flexibility and scalability in the installed reflectors. With this pervasive infrastructural intervention, it is reasonable to plan other generally useful features that could be symbiotically tied to IRS. An IRS can co-exist with very dense sensors which use the reflector for coding spatiotemporal information about measurements, updating information in real time and, if needed, integrate with other features of smart buildings.

Radio wave propagation and air circulation, while fundamentally different, share some common issues: complex indoor layouts present challenge for both ventilation and wireless coverage. Indoor scenarios envisioned for $6 \mathrm{G}$ applications of IRS [2] ask for mass deployment of IRS in such spaces; the public health authorities at the same time suggest that these environments are the hotbeds of viral particle concentration, and consequently represent locations where high levels of virus transmission take place. The coinciding maximal utility of both intelligent reflective surfaces and viral detectors hence suggest the advantage of joint deployment, which we explore in this paper.

Our proposed solution is a symbiotic integration of viral detectors (VD) with the IRS. The detection of viruses at indoor environments have been done using devices that require the sampling of the particles in the air, which can be a cumbersome process [5]. However, for the proposed Intelligent Reflector - Viral Detectors (IR-VD), adhesive strips with chemical receptors can capture virus particles that bind to the strips to perform detection. This solution can be applied to any indoor environment and help to quickly identify a possible airborne infection in that space.

Upon sensing virus particles that have bound to the strips, the unit cells will reflect signals with an embedded code to 


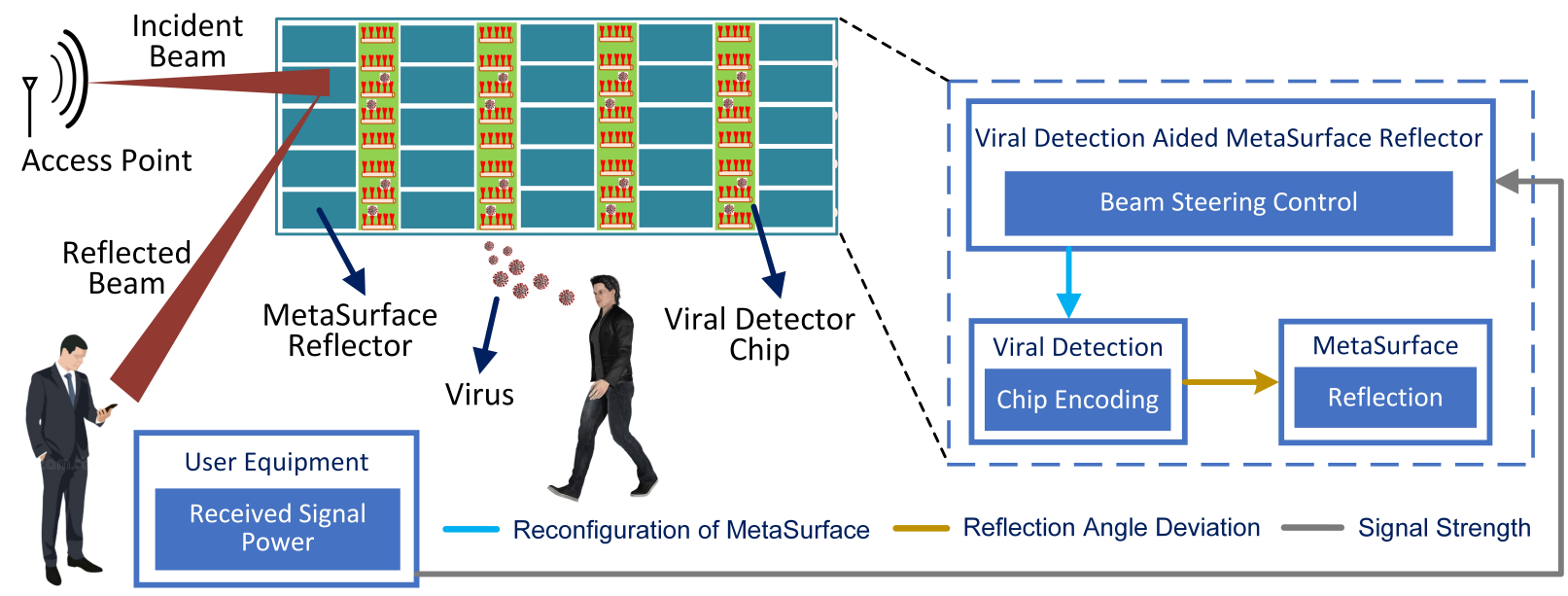

Fig. 1: Setup of Intelligent Reflector - Viral Detectors (IR-VD) with an array of patch antennas integrated with adhesive viral detector strips, as well as protocol modules for controlling the beam forming and manipulation for information encoding.

provide information about the virus, and comes in the form of phase manipulation that results in beam flicking encoding a binary string, which is detected at the receiver by sensing the power deviation of the signals. Patches that are not adjacent to such virus-affected areas will not have this embedded code. Once detected by the mobile device, the embedded code and its location will indicate the zone of the wall that is contaminated with the virus. The adhesive strips can be engineered to have detectors that are specific to a variety of virus types. The code that is embedded into the signals reflected from IR-VD can be specific to each virus type.

In this paper, we present the design of IR-VD, motivation for its development, and simulation of its performance which verifies the principle of operation and explains it practically. We give a contextual overview of viral detection, as well as the convergence of different technologies and means of communications that have enabled development of the concept, and discuss potential challenges and future work.

\section{AIRBORNE VIRUS BACKGROUND}

Indoor environments can expose humans to a variety of airborne viruses, some of which may cause respiratory diseases. These pathogens can be transmitted through air in the form of liquid droplets or airborne particles [6]. Due to their small sizes $(1-40 \mu \mathrm{m}$ when coughing) and low settling velocity (up to $3.1 \times 10^{-3} \mathrm{~m} / \mathrm{s}$ for $10 \mu \mathrm{m}$ particles), these viruses can remain in the air, increasing their likelihood of infecting other people within an indoor environment [5], [6]. The virus survivability in the air is also related to environmental factors, such as temperature, relative humidity $(\mathrm{RH})$ and surrounding biological materials that can absorb the virus [7]. In this case, virus survivability is related to the physical protection against environmental changes provided by the biological material (i.e., mucus) that encapsulates the viruses [7]. On the other hand, RH values affect the survivability of each virus family differently. For example, most respiratory viruses, including influenza, survive longer in environments with RH between $20 \%$ and $30 \%$. However, non-enveloped viruses (e.g., polio

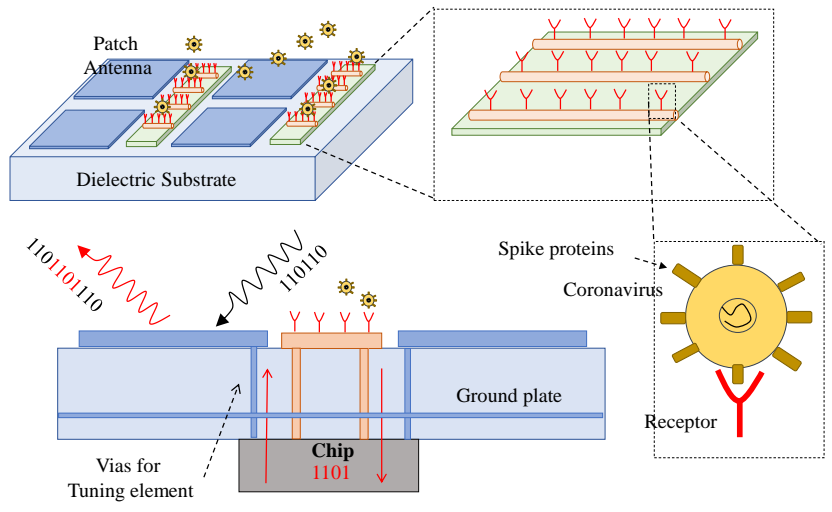

Fig. 2: Overview of the IR-VD, with an expanded view of the viral detector strip, showing the conductive tubes and the viral receptors. The viral receptor is applied on the surface of the conductive tubes to bind and detect the virus. The lateral projection of the intelligent reflector illustrates the generation and embedding of a binary code (highlighted in red) on the reflected electromagnetic wave whenever a virus is detected.

virus) show higher survivability at higher $\mathrm{RH}$ that is between $70 \%$ and $90 \%$ [7].

Viral and droplet/particle sizes are also related to the different infected areas of the respiratory tract. For example, when inhaled, larger particles (greater than $5 \mu \mathrm{m}$ ) deposit on the upper respiratory tract while smaller particles deposit in the lower part of the lungs and can cause severe pneumonia [6]. The infected people will then become the new source of viral aerosols and can spread them through coughing, sneezing or speaking when they are close to other people. In addition to these, there are other sources of viral aerosols in the home environments, such as flushing toilets (liquid droplets coming from the toilet can carry viral particles excreted from the human body) and changing diapers (by manipulating diapers some viral particles can be thrown in the air), and in laboratory settings, e.g. shaking, homogenisation and sonication of ma- 
terials that contain viral particles [6]. The campaign around social distancing can reduce viral aerosols and hence the propagation of virus to infect other people [5], [6]. Therefore, air quality has become a public health and safety concern due to growing numbers of respiratory infections, especially in recent years with the SARS and COVID-19 pandemics. This has driven investigation into new methods to improve airborne virus detection and identification [7]. In the next section, we introduce a new solution to monitor the presence of airborne viruses within an indoor environment, which can be applied for commercial or domestic purposes to help detect airborne as well as viral droplets.

\section{INTELLIGENT REFLECTORS FOR VIRAL DETECTION}

As described in the introduction, future wireless communication systems might use a new form of IRS infrastructure placed on the wall that are used to reflect high frequency spectrum signals to devices. Our proposed approach is to make use of this new infrastructure to detect viral particles, enabling it to have dual function: high-speed wireless communication and sensing airborne viral and communicating to mobile devices. This makes viral sensing and IRS a good match, since virus sensing can be integrated with other prominent wireless communication systems of the future, which is appealing from a deployment perspective as well as a future sensing technology: pervasive, reliable, high-tech entities that not only coexist, but support one another. In the context of proliferation incentives, they also form a symbiotic relationship: the push for better connectivity would bring health protection, and vice versa, contributing to wide market penetration. Mass deployment of IRS without sensing capabilities in that sense would be a wasted opportunity; mass deployment of viral detectors with specialised communication modules without the supporting role for wireless networks would be an improbable investment.

\section{A. Viral Detection Beam Steering Protocol}

IRS functionalities can be extended to detect airborne virus within an indoor environments by adding viral detector strips, illustrated in Fig. 2. The strips, combined with the components found in most IRS: patch electromagnetic antennas, a dielectric substrate, a ground metallic material, and a chip that controls the phase changes of the reflected signals, constitute the IR-VD. The adhesive viral detector strips will be placed in the spaces between the patch antenna tiles and contain functionalized receptors that correspond to the proteins on the virus membrane. These receptors can detect single or multiple families of viruses. For example, human $\alpha$-2,6-linked sialosaccharides have been reported to bind influenza virus family, making these sugars suitable candidates for the receptors [8]. In terms of integrating with the conductance change that interfaces to the chip, we can foresee the usage of electrostaticbased detection using protein-ligand response that changes the conductance in a substrate with response time around 2 minutes for viruses such as Influenza, A/HN1, H5N1 AND SARS [5].

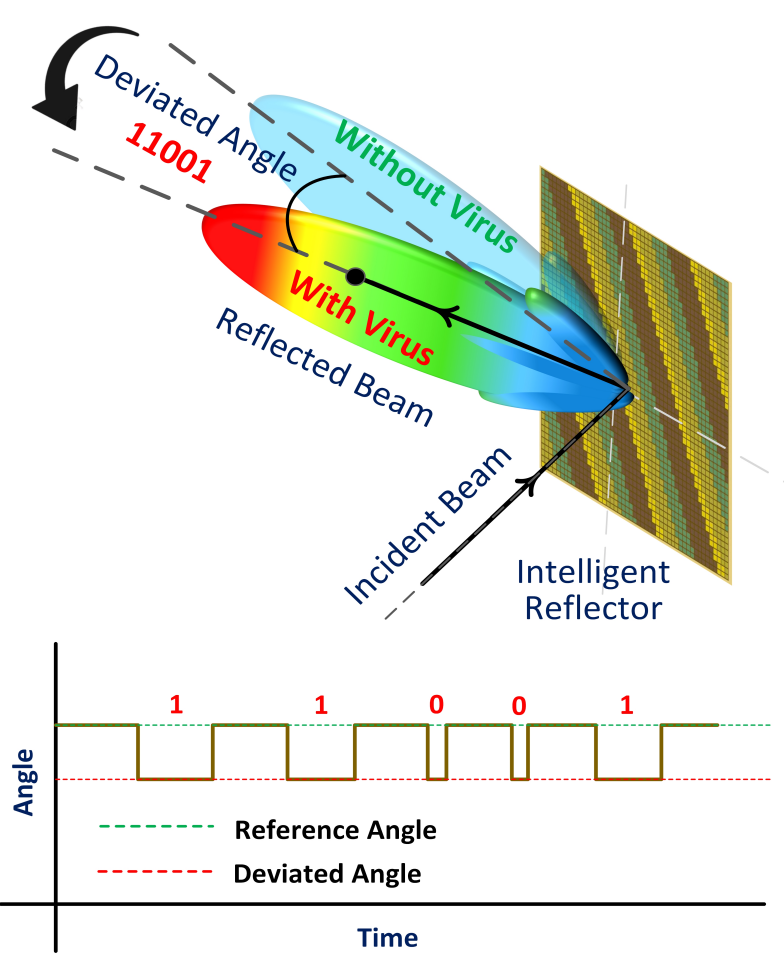

Fig. 3: The proposed approach for information encoding via phase change of the reflected beam. In absence of virus, the beam reflects towards the receiver, while presence of virus encodes the information by flicking the beam. Time variations between flicking of the beam generates the information bits about the presence, location and concentration of the virus.

This modular design will enable the providers to customise the reflectors to their needs, such as focusing on the detection of the most dangerous families of viruses, in case of a viral outbreak or pandemic. The process of viral detection and monitoring can be described as follows: upon viral contact with the adhesive receptor strips, the conductance along the tubes containing the receptors will change, indicating viral presence on a particular section of the IR-VD.

The change in conductance will be detected by the chip that controls the beam steering, and will encode information by flicking the phase change of the signals, as depicted in Fig. 3. The encoded information will depend on the duration between the phase changes (e.g., a digital 1 bit will result in a longer durations between the steered beams compared to digital 0 bit) [3]. Therefore, the flicking of the beams will need to be detected by the receiver, which will also need to detect the timing between the power changes as the beams are flicked. The encoding of the information can include the type of virus, as well as the density on the wall, and possibly the location on the IR-VD.

\section{B. Viral Detector Strips for IRS}

Since the introduction of IRS, a number of different solutions and configurations have been proposed: here we cite three IRS solutions based on their frequency of operation, tile size 
material used and steering granularity. Moreover, we describe the free spacing on the tile (i.e., space that is not occupied by the patch antenna or other electronic device), and the density of viral detectors that can be placed on the tile depending on this free spacing. In [9], a novel antenna structure is proposed to individually control the phase of the reflected signal by configuring different RF paths at 2.3 and $28.5 \mathrm{GHz}$ with steering capabilities of $-60^{\circ}$ to $+60^{\circ}$. Each tile is composed of three reflective paths arranged in a $\mathrm{T}$-shaped configuration with a fourth dummy path just to maintain a symmetric structure. For this solution, the antenna patch is placed in the center of the IRS tile $\left(50 \times 50 \mathrm{~mm}^{2}\right)$, and the following spaces are free and can be used to place the viral detectors: $13 \times 50 \mathrm{~mm}^{2}$ for the left and right sides, and $13 \times 37 \mathrm{~mm}^{2}$ for the upper and lower sides. Based on these areas dimensions, approximately 565 viral detectors can be placed in a single tile.

A similar structure was proposed in [10], where an IRS was designed to recognize human gesture and respiration in real-time by transmitting and receiving electromagnetic waves and processing them using an artificial neural network. This solution has plenty of open areas around the antenna patch on the $54 \times 54 \mathrm{~mm}^{2}$ tile where the viral detectors can be placed at: $17 \times 54 \mathrm{~mm}^{2}$ on the left side, $17 \times 37 \mathrm{~mm}^{2}$ the upper side, $17 \times 36 \mathrm{~mm}^{2}$ on the lower side (due to the presence of an inductor on the IRS), $17 \times 17.4 \mathrm{~mm}^{2}$ and $17 \times 26.6 \mathrm{~mm}^{2}$ on the right side (above and below the resistor present on the IRS, respectively). Given the spacing on each tile, this model is able to fill a higher quantity of viral detectors, reaching up to 726 per tile.

Other designs can be considered as well, however they might not posses enough free spacing to place a great number of viral detectors as the squared shape with the centered patched antenna described in [9], [10]. For example, we also reviewed the design proposed by [11]. where small tubes filled with water are used to reflect electromagnetic waves with steering capabilities of $-20^{\circ}$ to $+20^{\circ}$. This IRS achieves different beam phases through a combination of empty and water-filled tubes for the reflectors with $20 \times 20 \mathrm{~mm}^{2}$ tiles. In this case, the free spacing are two small areas of $16 \times 6 \mathrm{~mm}^{2}$, so 48 viral detectors can be placed.

\section{Simulations}

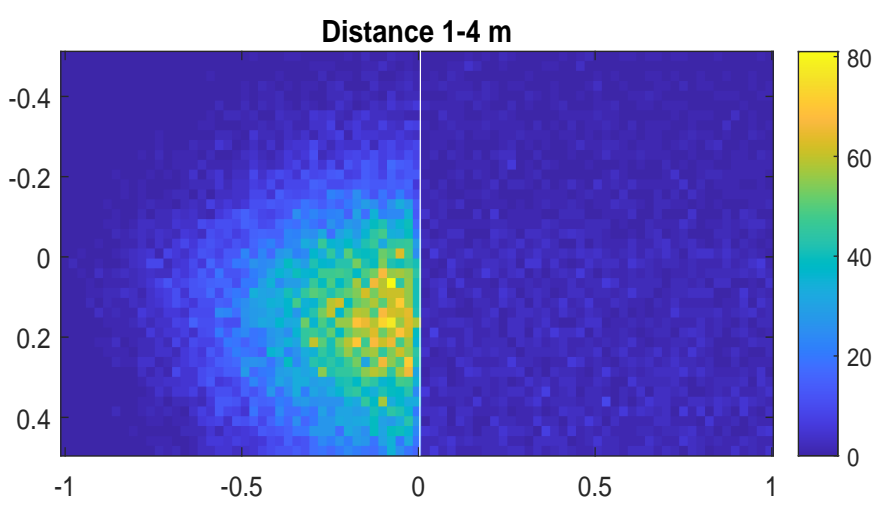

Fig. 4: Simulated viral density in $1 \mathrm{~m}$ (left) and $4 \mathrm{~m}$ (right) simulations
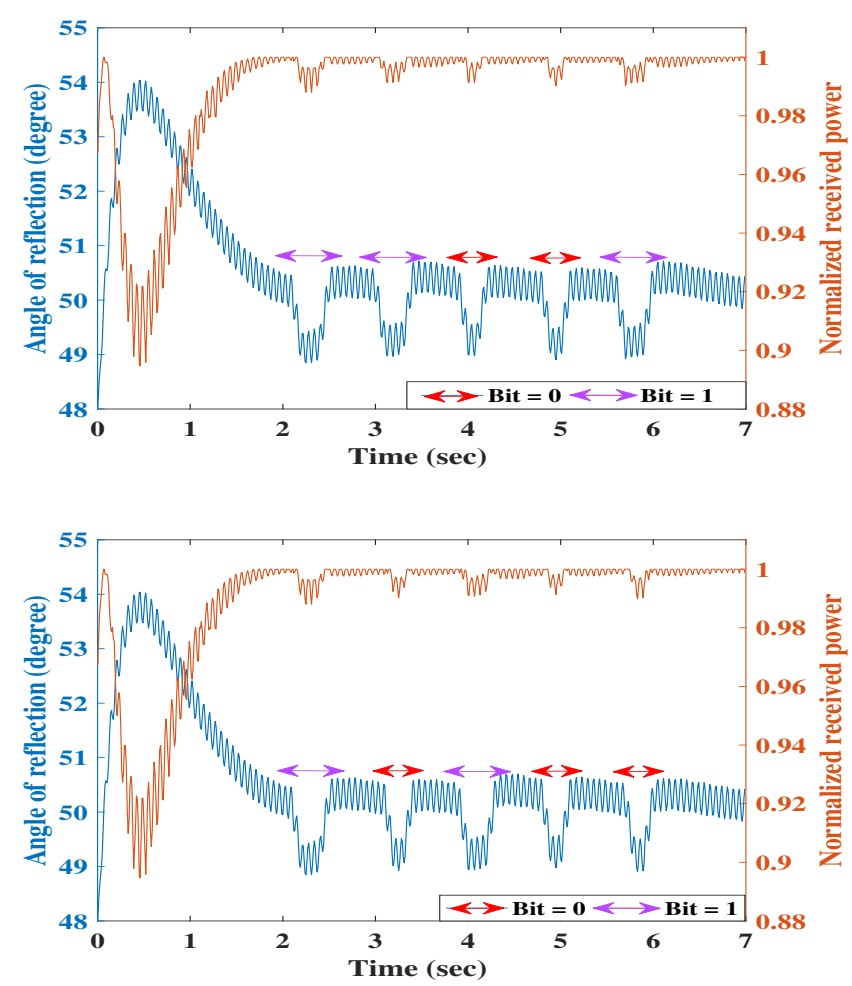

Fig. 5: Observed angles of reflection and normalized received signal power for $1 \mathrm{~m}$ (top) and $4 \mathrm{~m}$ (bottom) simulation

\section{A. Simulation Scenario}

In the scenario shown in Fig. 1, the people inside the room disperse droplets with viral particles (e.g., by sneezing), and the droplets that bind to the portion of the wall occupied by our IR-VD is of interest to us. This scenario setting can be interpreted through the concept of an breath molecular communication model [12].

In a breath molecular communication system, we observe the dispersion of aerosol from the respiratory tract of the people present in the space. The droplets with viral content that reaches the IR-VD and binds onto the surface will trigger a message, which is created and encoded into the reflected beam that will flick depending on the data to be transmitted. If observed as a molecular MIMO (multiple input, multiple output) system, with people as viral transmitters, and different IR-VD strips as the receivers, signal detection would allow identification of sources based on received signal even if the sources are not spatially distanced. While such an option opens the question of anonymity if identification is done at the level of an individual, it is not hard to devise a use-case in which such information is used through aggregation. For example, if the patches are placed within an air conditioning network serving several rooms at a workplace, knowing which room viral air stream originates from is useful.

\section{B. Viral Molecular Communication and Binding Model}

For the simulated effects of molecular communication, sneezing (quick burst transmission in the atmospheric channel towards a dense array of receivers on the wall), the effects of 
the channel can be modelled in a relatively simple manner. A range of droplets $(50,000)$ of relatively large mass (mean $10^{-4} \mathrm{~g}$, standard deviation $10^{-4} \mathrm{~g}$ ) originating from the same point in space under a range of horizontal (mean $0^{\circ}$, standard deviation $18^{\circ}$ ) and vertical (mean $-6^{\circ}$, standard deviation $12^{\circ}$ ) angles and initial velocities (mean $11.2 \mathrm{~m} / \mathrm{s}$, standard deviation $3 \mathrm{~m} / \mathrm{s}$ ) travel as projectiles with air drag effects towards the wall. With breathing or speaking, the effects of the dynamic molecular communication channel become more emphasised, with the air movement dictating the motion of small particles with low initial velocities. Once the particles reach the IR-VD, the density of the receptors per tile (in our case 160) dictates the sensing capabilities and the resolution of measurement.

The simulated virus binding process is based on the model previously reported in [13]. A chemical reaction occurs whenever the virus proteins, i.e. ligands, have physical contact with the host receptors, which in this case they will be the viral detectors, resulting in the binding of these proteins. Strong attachment is a design requirement to ensure that the virus will remain bound to the viral detector, and it will be dependent on the concentration of viral proteins and host receptors on the adhesive strips, which depends on the type of IRS discussed in Section III (b). These concentrations are estimated using the ligands, receptors, virus and viral detector dimensions found in [13].

\section{IRS Simulator}

We have performed feedback-based beam steering mechanism simulations on Matlab to demonstrate the performance of our proposed method. More specifically, we have used an Extremum Seeking Control (ESC) algorithm [3] to steer the beam towards the receiver. In this scheme, a dither signal (of 0.5 amplitude and $50 \mathrm{rad} / \mathrm{s}$ frequency in our case) is used to excite the periodic response of the received signal power and corresponding change in the received power is used to drive and maintain the angle of reflection. For more details, readers are referred to [3]. Once the virus and its concentration have been detected by viral detection strips, this information is conveyed to the receiver by deviating the beam from its desired angle while at the same time, the beam steering mechanism tends to bring the beam back to its desired angle.

\section{Simulation Results}

As indicated in Fig. 3, the information about virus detection via distributed sensors on IR-VD is encoded into its reflection properties. This results in the deviation of the beam at the receiver, which gets notified about the presence of virus by decoding the pattern given by the changes occurring to its received signal power. Fig. 4 is a combined rendition of simulated particle dispersion over a $1 \times 2 \mathrm{~m}$ IR-VD placed on the wall with a varying distance of the sneezing person standing in front of the surface. The left half of the IR-VD represents the effect of $1 \mathrm{~m}$ distance, and the right represents the $4 \mathrm{~m}$ distance. The heat map colour represents the density of droplets on the surface as the number of positive detections per tile.
When the virus information encoding process is integrated with our beam steering scheme [3], IR-VD communicates information about viral contamination. In Fig. 5 we observe that, after reflecting through the IR-VD, the beam was initially directed towards $48^{\circ}$ but the receiver was located at $50^{\circ}$ (the reference angle) with respect to the wall. Our beam steering algorithm works based on a feedback principle and tends to steer the beam towards 50 degrees at receiver location with short transient flicking behavior of around 1 second. It can be observed that once the beam tracks the receiver at $50^{\circ}$, at the time instant ( $1 \mathrm{~s})$, the received signal power is at its maximum normalized value of 1 . At $2 \mathrm{~s}$ time instant, the beam flicking process starts due to the viral detection. The flicking results in a small angle deviation based on the phase gradient change. This disturbance causes the angle to deviate by 1 degree and the beam reflects towards $49^{\circ}$, and there is a corresponding decrease in the received power at the same instant. The pattern of change in the received signal power and the timing between the beam flicks, conveys a message to the receiver. Indicatively, we have considered 5 bits of information (5 deviations) which are encoded at 2.5, 3.1, 4.1, 5.1 and 6.1 seconds. The first two bits are used to represent the concentration and the type of virus detected, while the last three bits correspond to the density of virus that has bound onto the IR-VD. The time duration of the deviation corresponds to bit 0 or 1 as shown in the figures as well. For the $1 \mathrm{~m}$ case, the width of the disturbance at 2.1, 3.1 and $6.1 \mathrm{~s}$ is double, corresponding to bit 1, compared to the time width of deviation at 4.1 and 5.1 second time instants, which corresponds to bit 0 .

\section{Challenges and Future Directions}

While the introduction of IRS will transform the future management of wireless signals, the integration of viral detectors with communication infrastructure to convey that information to mobile devices will provide even more motivation for investing in such infrastructure.

However, there are a number of challenges that will need to be overcome for IR-VD to have its dual function. This section will review some of these challenges and future directions.

\section{A. Viral Detection Accuracy}

Airborne virus detection is particularly challenging since it aims to obtain a sample of the viral particles to detect. Existing literature [5] shows that these techniques need to consider fundamental system characteristics including particle random motion propagation, sample quantity variations on the reflector that can be due to low viral particle density in the aerosol particles, dust interference, and the affinity of virus binding to receptor structures on the adhesive strips. These challenges currently inhibit developments in airborne virus detectors, which is reflected in the number of existing products available in the market today. At the same time, suitable local air-flow is needed to ensure that the virus can bind successfully onto the receptors and to protect the sample for the duration of the detection. 
The usage of air trapping mechanisms can protect the air samples in the vicinity of the biosensing technology

However, the design of the intelligent reflectors, whether they have different sizes and shapes of tiles, can possibly overcome these challenges, and further mathematical and testing work is most needed.

Tiles with dimensions we surveyed in section III-B have a total area in the order of magnitude of the human head orifices capable of taking in a respiratory virus. The number of viral detectors on each tile is at the order of magnitude of viral particle count in the minimal infectious dose (hundreds) and allows detection of sub-minimal doses. Hence, the probability of a single tile detecting presence of the virus in an indoor environment is greater than that of a single individual being infected. We multiply this probability by the number of deployed tiles, which by far exceeds the number of people in the indoor space under observation. Linking the probability with the expected time of detection, this means that our system detects the virus much faster than the circulating virus would infect a susceptible person in the space of interest.

\section{B. Radio environment challenges}

Our presented coding mechanism causes small received power fluctuations. To distinguish these from noise or the mobility effects, we need to consider robust coding schemes, as well as power thresholds that can realistically be used in such applications. This opens another strand of our future work, which would employ both study of the RF channel and methods of artificial intelligence for the detection of the beam flicking.

\section{Virus Ultraviolet Treatment}

While the detectors can enable the virus to bind to provide information on hazardous regions, there also needs to be a solution to treat the virus to minimize physical contacts between people and the wall. Recent research has found that using ultraviolet light can inactivate airborne viruses [14]. A possible addition to the adhesive strips are local ultraviolet sources that are able to inactivate the virus once they have bound onto the receptors. The frequency range of the ultraviolet light are chosen so that it only affects the viral proteins and not the receptors on the strip. This ensures that the strips can be reused again to allow the receptors to become free, and at the same time ensure that the droplets do not infect any people within the vicinity. However, this can increase the complexity and cost of the IR-VD in order to ensure that the ultraviolet source will emit sufficient rays to treat the bound virus.

\section{Multi-beam consideration}

In this paper, we have considered a case of single beam, where a single incident beam after reflecting via the IRS is steered towards a single receiver. However, when the proposed approach is applied for the case of multiple-beams or multipath communication, additional phase-shifts incurred by the viral information may cause interference. We intend to address this in our future work, where our objective will be to apply the phase gradients in such a way to jointly maximize the received signal power and to minimize the phase difference among multi-beams to have constructive interference.

\section{E. Integration into the Environment}

A limitation of the proposed solution is that it will only detect the virus emitted through droplets in the vicinity of the reflectors. A next step would be the application of the viral detector strips to infrastructures within domestic and commercial environments, such as tables, floors and shopping store shelves. For that we need higher complexity beam control techniques that can overcome also signal losses from different object materials, such as the one proposed in [15] for example. This will ensure very accurate detection of any virus droplets in areas of high density of people. However, challenges need to be considered. One is the task of transmitting signals from the strips to a device within the vicinity, which may require reflection of signals from a base station. Another is the increase in cost of designing furniture, cabinets, and shelves that contain integrated circuits to connect to the adhesive strips containing the virus detectors as well as the reflection communication system.

\section{CONCLUSION}

Novel air-borne viruses can bring modern civilisation to an abrupt halt, as shown by the COVID-19 pandemic. In indoor environments, viral particles are often deposited on surfaces, easily landing there when virus carriers breathe, sneeze, cough or speak. Notification of such particles on surfaces in the room would be helpful information for health and safety protocols in the building, for people in the shared common space, and for general public health. Our design of IR-VD enables such pervasive sensing of viral presence, coupled with the rollout of next generation wireless infrastructure, in the form of IRS. The paper presents IR-VD, which integrates such viral detectors through adhesive strips and how they can be integrated into conventional IRS. The communication of the viral load is achieved by manipulating the reflected signals through beam flicking that encodes the information about the virus bound on the wall, while not compromising the original reflected signals. We have shown how such a system could operate, why the convergence of technologies in this case is natural and necessary, and what the future directions might look like for this synergy of communications and health technology, which is very much in the spirit of the sixth wireless generation.

\section{REFERENCES}

[1] V. Ziegler and S. Yrjola, "6G indicators of value and performance," in 2020 2nd $6 G$ Wireless Summit (6G SUMMIT). IEEE, 2020, pp. 1-5.

[2] R. Alghamdi, R. Alhadrami, D. Alhothali, H. Almorad, A. Faisal, S. Helal, R. Shalabi, R. Asfour, N. Hammad, A. Shams, N. Saeed, H. Dahrouj, T. Y. Al-Naffouri, and M. S. Alouini, "Intelligent surfaces for 6G wireless networks: A survey of optimization and performance analysis techniques," IEEE Access, vol. 8, pp. 202 795-202 818, 2020.

[3] N. Ashraf, M. Lestas, T. Saeed, H. Taghvaee, S. Abadal, A. Pitsillides, and C. Liaskos, "Extremum seeking control for beam steering using hypersurfaces," in 2020 IEEE International Conference on Communications Workshops (ICC Workshops). IEEE, 2020, pp. 1-6. 
[4] X. Lu, E. Hossain, T. Shafique, S. Feng, H. Jiang, and D. Niyato, "Intelligent reflecting surface enabled covert communications in wireless networks," IEEE Network, vol. 34, no. 5, pp. 148-155, 2020.

[5] C. F. Fronczek and J.-Y. Yoon, "Biosensors for monitoring airborne pathogens," Journal of laboratory automation, vol. 20, no. 4, pp. 390 410, 2015.

[6] S. A. Sattar, N. Bhardwaj, and M. K. Ijaz, Airborne Viruses. American Society for Microbiology Press, 2007, ch. 3.2.7, pp. 1-21.

[7] M. K. Ijaz, B. Zargar, K. E. Wright, J. R. Rubino, and S. A. Sattar, "Generic aspects of the airborne spread of human pathogens indoors and emerging air decontamination technologies," American Journal of Infection Control, vol. 44, no. 9, pp. S109-S120, 2016.

[8] M. de Graaf and R. A. Fouchier, "Role of receptor binding specificity in influenza a virus transmission and pathogenesis," The EMBO journal, vol. 33, no. 8, pp. 823-841, 2014.

[9] L. Dai, B. Wang, M. Wang, X. Yang, J. Tan, S. Bi, S. Xu, F. Yang, Z. Chen, M. Di Renzo et al., "Reconfigurable intelligent surface-based wireless communications: Antenna design, prototyping, and experimental results," IEEE Access, vol. 8, pp. 45913-45 923, 2020.

[10] L. Li, Y. Shuang, Q. Ma, H. Li, H. Zhao, M. Wei, C. Liu, C. Hao, C.W. Qiu, and T. J. Cui, "Intelligent metasurface imager and recognizer," Light: Science \& Applications, vol. 8, no. 1, pp. 1-9, 2019.

[11] A. H. Naqvi and S. Lim, "A beam-steering antenna with a fluidically programmable metasurface," IEEE Transactions on Antennas and Propagation, vol. 67, no. 6, pp. 3704-3711, 2019.

[12] M. Khalid, O. Amin, S. Ahmed, B. Shihada, and M.-S. Alouini, "Communication through breath: Aerosol transmission," IEEE Cоттиnications Magazine, vol. 57, no. 2, pp. 33-39, 2019.

[13] D. P. Martins, M. T. Barros, M. Pierobon, M. Kandhavelu, S. Balasubramaniam et al., "Computational models for trapping ebola virus using engineered bacteria," IEEE/ACM Transactions on Computational Biology and Bioinformatics, vol. 15, no. 6, pp. 2017-2027, 2018.

[14] D. Welch, M. Buonanno, V. Grilj, I. Shuryak, C. Crickmore, A. W. Bigelow, G. Randers-Pehrson, G. W. Johnson, and D. J. Brenner, "FarUVC light: A new tool to control the spread of airborne-mediated microbial diseases," Scientific Reports, vol. 8, no. 1, pp. 1-7, 2018.

[15] M. Pengnoo, M. T. Barros, L. Wuttisittikulkij, B. Butler, A. Davy, and S. Balasubramaniam, "Digital twin for metasurface reflector management in $6 \mathrm{~g}$ terahertz communications," IEEE Access, vol. 8, pp. 114580 $114596,2020$.

Harun Šiljak (M'15, SM'20) graduated from the Automatic Control and Electronics Department, University of Sarajevo (B.E. 2010, M.E. 2012) and the International Burch University Sarajevo (Ph.D. 2015). He is an assistant professor in embedded systems at Trinity College Dublin, Ireland, working on complex systems and non-conventional computation, communications and networks.

Nouman Ashraf (STM'15) received the PhD degree in electrical engineering from Frederick University, Cyprus, under Erasmus Mundus Program. He received the M.S. and B.S. degrees in electrical engineering from COMSATS University, Pakistan. His research interests include application of control theory for algorithms designing in emerging intelligent networks.

Michael Taynnan Barros (M'16) is a Lecturer at the School of Computer Science and Electronic Engineering, University of Essex, UK, and a Marie Skłodowska-Curie Individual Fellow at the BioMediTech Institute of the Tampere University, Finland. He received his $\mathrm{PhD}$ in Telecommunication Software at the Waterford Institute of Technology, Ireland, in 2016.

Daniel Perez Martins (STM'05, M'19) is a Postdoctoral Researcher and the Technical Lead of the Biomedical Nano and Molecular Telecommunications Team at Walton Institute. His research concentrates on the modeling and analysis of conventional and nanoscale communications systems. He received his $\mathrm{PhD}$ from the Waterford Institute of Technology, Ireland, in 2019.
Bernard Butler (STM'10, M'17) was awarded BSc and MSc by University College Cork and $\mathrm{PhD}$ by Waterford Institute of Technology (WIT). He is a senior research fellow at the CONNECT Centre, WIT. His research interests include modelling the performance and security of complex systems, especially those related to networking beyond $5 \mathrm{G}$.

Arman Farhang (M'13) is an Assistant Professor at Maynooth University, Ireland. He received the $\mathrm{PhD}$ degree in 2016 from Trinity College Dublin, Ireland where he was a research fellow until September 2018, when he joined Maynooth University. His research interests include digital signal processing for communications, multiuser, multiantenna, and multicarrier systems.

Nicola Marchetti (M'13, SM'15) is Associate Professor at Trinity College Dublin. He received the $\mathrm{PhD}$ in Wireless Communications from Aalborg University in 2007, the M.Sc. in Electronic Engineering in 2003, and the M.Sc. in Mathematics in 2010. His research interests include radio resource management, self-organising networks, and signal processing.

Sasitharan Balasubramaniam received his Bachelor (electrical and electronic engineering) and Ph.D.degrees from the University of Queensland in 1998 and 2005, respectively, and his Master's (computer and communication engineering) degree in 1999 from Queensland University of Technology. His current research interests include the Internet of Nano Things and molecular communication. 